\title{
Review
}

\section{The Nishino Breathing Method and Ki-energy (Life-energy): A Challenge to Traditional Scientific Thinking}

\section{S. Tsuyoshi Ohnishi ${ }^{1, *}$ and Tomoko Ohnishi ${ }^{2}$}

\author{
${ }^{1}$ Philadelphia Biomedical Research Institute, King of Prussia, PA 19406, USA and ${ }^{2}$ Department of Biochemistry \\ and Biophysics, University of Pennsylvania School of Medicine, Philadelphia, PA 19104, USA
}

\begin{abstract}
The breathing method, which was developed and is being taught by Kozo Nishino, a Japanese Ki-expert, is for raising the levels of Ki-energy (life-energy or the vitality) of an individual. It is neither a therapy nor a healing technique. However, many of his students have experienced an improvement in their health, and in some cases, they were able to overcome health problems by themselves. Since this is an interesting subject from the standpoint of complementary and alternative medicine (CAM), we have been collaborating with Nishino to conduct a scientific investigation of his Ki-energy. We found that Nishino's Ki-energy can inhibit cell division of cancer cells, protect isolated mitochondria from heat deterioration and reduce lipid peroxidation in heat-treated mitochondria. Although Ki-energy may consist of several different energy forms, we found that at least one of them is near-infrared radiation between the wavelength range of 0.8 and $2.7 \mu \mathrm{m}$. Another interesting observation at his school is the Taiki-practice (paired Ki-practice). During this practice, Nishino can 'move' his students without any physical contact. Many of them run, jump or roll on the floor when they receive his Ki-energy. We studied this and propose that 'information' is conveyed through the air between two individuals by Ki-energy. This may be called a five sense-independent, life-to-life communication by Ki. All of our results suggest that we should re-evaluate the Cartesian dualism (separation of mind and body) which has been a fundamental principle of modern science for the past three centuries.
\end{abstract}

Keywords: Nishino Breathing Method - Ki-energy - infrared radiation - inhibition of cultured cancer cell division - Taiki-practice - five sense-independent communication - Ki-information entropy - Cartesian dualism - biological Uncertainty Principle

\section{Introduction}

Modern science advanced out of the 'Dark' ages as it began to accept the 'separation of mind and body.' Descartes proposed that the movement of an object (e.g. a canon ball) can be perfectly described by mathematical equations, and that the trajectory is not influenced by the human mind (Cartesian dichotomy). This mathematical and mechanical worldview, which was pioneered by Galileo and Descartes, and fully developed by Newton, has been tremendously successful and

*For reprints and all correspondence: S. Tsuyoshi Ohnishi, Philadelphia Biomedical Research Institute, 100 Ross and Royal Road, King of Prussia, PA 19406-0227, USA. Tel: +1-610-688-6276; Fax: +1-610-254-9332;

E-mail: stohnishi@aol.com has led to the development of the material worldview of the 20th century.

Newtonian physics, which was based upon the concepts of absolute time and space, succeeded in predicting the occurrences of solar and lunar eclipses over several thousand years with amazing accuracy. It also succeeded in calculating the trajectory of comets. However, his concepts of absolute time and space were denied by Einstein's theory of relativity. Newton's worldview guarantees that the velocity and position of any object can be determined at any time. This seemingly natural assumption is not valid at the atomic level. According to Heisenberg's 'Uncertainty Principle', the velocity and the position of an electron (or any atomic particle) cannot be determined simultaneously. When an observer measured the

(C) The Author (2006). Published by Oxford University Press. All rights reserved.

The online version of this article has been published under an open access model. Users are entitled to use, reproduce, disseminate, or display the open access version of this article for non-commercial purposes provided that: the original authorship is properly and fully attributed; the Journal and Oxford University Press are attributed as the original place of publication with the correct citation details given; if an article is subsequently reproduced or disseminated not in its entirety but only in part or as a derivative work this must be clearly indicated. For commercial re-use, please contact journals.permissions@oxfordjournals.org 
electron position, then its velocity would be influenced by the measurement and became uncertain.

These new developments in physics in the 20th century made the use of nuclear energy possible. The appearance of atomic bombs, hydrogen bombs and nuclear power plants completely changed the political, economic and cultural atmosphere of the 20th century. However, Newtonian physics seemed to have survived through the 20th century despite the drastic changes in our scientific understanding. The reason may be that relativistic physics plays a significant role only in systems, which move at a velocity close to that of light, and the Uncertainty Principle is only observed at the atomic level. Therefore, in our daily life, Newtonian worldview still seems to be valid. As to the Cartesian dichotomy, this seems to be quite acceptable in our material worldview.

Only modern medical views have begun to recognize the importance of the mind-body relationship and are questioning Descartes's original idea of the separation of mind and body (Cartesian dualism) in dealing with human beings. However, this idea still pervades modern civilization and culture in the western hemisphere.

Another fundamental concept in modern science is that experimental results should be reproducible. In other words, the results should be independent of where, when and by whom the experiment is performed. Otherwise, the result cannot have validity.

Now, in this article, we would like to present several observations that Ki-energy may provide a good example to examine and re-evaluate complex questions, such as 'To what extent are mind and body correlated within one person?' 'Can the mind of one person directly affect the mind of others without using the five senses?' 'Can the mind of one person affect the physical condition of others?' Or 'Is an observer a part of an experimental system?'

\section{What is the Nishino Breathing Method?}

Kozo Nishino, who is one of the leading Ki-experts in Japan, studied medicine, and then studied ballet at the Metropolitan Opera Ballet School in New York. After coming back to Japan, he founded the Nishino Ballet Company, through which he presented many classical and modern ballets as a director and choreographer. At the age of 50, he decided to search for the secrets of the Japanese martial art called Aikido. He quickly became a 7 th degree blackbelt and trained many students as an Aikido master. Combining all of his experiences, namely western medicine, western ballet and Japanese martial art, and through his continuous search for the secrets and beauty of the human body, he developed the Nishino Breathing Method. He developed his method independently from the Chinese Qigong practice.

Nishino method consists of relaxing, stretching, twisting and/or rotating the body combined with a slow, deep breath (one cycle of breath in 1-2 min) (1,2). One of his unique methods is called 'Taiki-practice' (literally meaning 'a paired
Ki-practice'). Both an instructor and a student extend hands and touch each other. The student sends $\mathrm{Ki}$ through his/her hand toward the instructor, and then the instructor sends back his $\mathrm{Ki}$ toward the student. They repeat the motion several times. He found that through this Taiki-practice, students can quickly develop their level of $\mathrm{Ki}$. He considers this to be a Ki-induced 'non-verbal' life-to-life communication without using the five senses. When he sends $\mathrm{Ki}$ to his students, many of them move, run, jump, dance or sing as if they are controlled by his instructions.

He has been teaching the method to more than 10000 students in Japan over the past 20 years (his publication list can be found in ref. (3)). Although Nishino does not offer any healing or therapeutic treatment, the practitioners have experienced beneficial health effects, for example, improvements in symptoms, such as high blood pressure, osteoporosis, arthritis and cancer. Perhaps, the most usual effect among practitioners is that everybody stays mentally and physically young and healthy. A study demonstrated that his method increased the immune activity of the practitioners and lowered stress levels (4). His Ki was shown to inhibit cell division of cultured human liver carcinoma cells (3). These results suggest that the Nishino Breathing Method is capable of improving our health.

He has hypothesized for many years that the breathing method would increase oxygen delivery in the body, activate cell metabolism including mitochondrial function, thereby bringing us tangible health benefits $(5,6)$. Recently, we found that his $\mathrm{Ki}$ protected isolated rat liver mitochondria from heat-induced deterioration, possibly by reducing the production of reactive oxygen species (ROS) (7). The protection of mitochondria and the reduction of ROS generation would produce more energy from the nutrients and would result in healthier cells and organs. The protection of mitochondria from adverse effects of ROS would reduce the likelihood of premature apoptosis, and therefore would contribute to the longevity of the practitioners. His prediction that mitochondria would play key roles in maintaining health and longevity seems to be supported by these experiments.

\section{Can His Students Emit Ki?}

One of the important criteria required to accept the Nishino Breathing Method as science is its universality. It is critical to prove that his students can also emit Ki. If only Nishino can display all these interesting phenomena but his students cannot, then the method would still remain in the realm of the paranormal and para-psychological phenomena. In order to test this, one of the authors (STO; practising this method on-and-off from 1997) examined his own ability to inhibit cancer cell growth in 2004 while we were collaborating with Tsurusaki and Yamaguchi (3). As shown in Fig. 1, STO could inhibit the growth of cultured cancer cells (HepG2) by emitting his $\mathrm{Ki}$ directly to the cultured cells. However, the degree of inhibition was less than Nishino's. When the culture dish was 


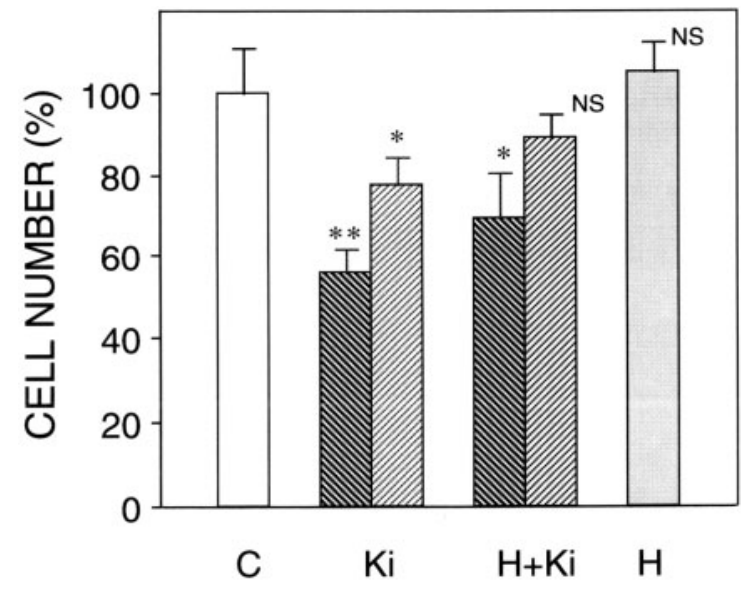

Figure 1. Cell numbers of cultured $\mathrm{HepG} 2$ cells counted $24 \mathrm{~h}$ after the culture dishes were exposed to Ki of Nishino (KN) and to one of the authors (STO) for $5 \mathrm{~min}$. Numbers are shown as percentages of the control (no Ki-exposed). Abbreviations are $\mathrm{C}$, control (no Ki-exposure); $\mathrm{Ki}$, left column is for cells exposed to KN's Ki and right column is for cells exposed to STO's; $\mathrm{H}+\mathrm{Ki}$, left column is for the cells exposed to KN's Ki through interrupting hands and right column is for the cells similarly exposed to STO's; H, interrupting hands alone. The error bars represent the standard error calculated from three measurements. *indicates that the significance of difference from the control was $P<0.05$ and $* * P<0.01$. NS indicates that the difference from the control is not significant. For details, see reference (3).

covered by the hands of another individual who did not practice the Nishino Breathing Method (see Fig 1B of the paper by Ohnishi et al. (3)), Ki from STO's fingers did not inhibit cell division. The difference from the control was not significant (as shown by NS in the figure). This may be explained as follows: (i) Since the cancer cells spread thinly on the bottom of the culture dish, STO's Ki could still reach the cells to inhibit cell division; (ii) However, if his $\mathrm{Ki}$ was interrupted by the hands of another person, his ability to inhibit cancer cell growth by penetrating other human tissue was limited. This is because his $\mathrm{Ki}$ was not as strong as Nishino's.

\section{Can His Student 'Move' Other Individuals by Ki?}

The most interesting scene in Nishino's class is to watch how he can move many of his students at his will during the Taiki-practice. When he sends $\mathrm{Ki}$ to students, they exhibit vigorous movements (run, jump or roll on the floor). Sometimes they dance or sing. Since this is such an extraordinary scene, many get an impression that this must be a trick. Some insist that this must be a kind of hypnosis, and others tend to believe that this is a paranormal phenomenon. Not only Nishino, but also dozens of instructors at his school can induce various movements in their students in Taikipractice, although the degree and the variety of movements are less.

Actually, a martial art technique called 'Toh-Ate' (literally meaning 'hit from a distance') has been known in Japanese martial art history as a secret technique to knock down the opponent from a distance without physical contact. Besides Nishino and the instructors at his school, Hiroyuki Aoki teaches a similar technique at his Shintaido School. He demonstrated his Toh-Ate technique at the 1984 JapanFrance Symposium on 'Science, Technology and Spiritual World' held at Tsukuba $(8,9)$.

In order to demonstrate that the Taiki-practice is neither hypnosis nor a paranormal phenomenon but a normal phenomenon, STO experimented whether he could move volunteer students of the Nishino School without physical contact. The protocol of the project was approved by the Institutional Review Board of the Philadelphia Biomedical Research Institute. The participants read the protocol and signed the consent form by agreeing that their photo may be published with their face partially covered to hide the identity. Fig. 2 shows that students (Y, F and M; whose eyes were covered by a blinder and ears were plugged by earplugs) stepped backward and fell down when he/she received Ki from STO. Since both their eyesight and hearing were blocked, this cannot be regarded as a hypnotic effect.

Although it was not as spectacular as what Nishino or other instructors demonstrate everyday in classes of Nishino's school, a student was still able to do this to a limited degree. A point to be emphasized is that STO can do this for only several students who are very 'sensitive' to $\mathrm{Ki}$, whereas Nishino and the instructors can do this for hundreds and thousands of students. Now the question comes as to what is the nature of $\mathrm{Ki}$ ?

\section{Near-Infrared Radiation Is also Involved in the Taiki-Practice}

As to the nature of $\mathrm{Ki}$, Chinese and Japanese scientists have already reported that it involves infrared radiation $(8,10-12)$. It was also reported that other forms of energy may be involved in $\mathrm{Ki}$ which include electromagnetic waves, electrostatic energy, magnetic energy and low frequency sound waves $(8,12,13)$. In our cultured cancer cell study (3), we proposed that infrared radiation may be a component of $\mathrm{Ki}$. In our mitochondrial study, we used visible range filters $(360-760 \mathrm{~nm})$ and near-infrared range filters (from 0.8 to $2.7 \mu \mathrm{m}$ ) to demonstrate that at least near-infrared radiation was involved in the observed Ki-effect (7). In preparing for this Review article, we tested whether near-infrared radiation was also involved in the Taiki-practice. We prepared a black vinyl curtain (size of $2 \mathrm{~m} \times 2 \mathrm{~m}$; thickness of $0.5 \mathrm{~mm}$ ) with a black acrylic plate $(30 \mathrm{~cm} \times 30 \mathrm{~cm}$; thickness of $3 \mathrm{~mm}$ ) attached (Fig. 3). To the acrylic plate, a window $(9.5 \mathrm{~cm} \times 12 \mathrm{~cm})$ was made on which another black acrylic plate (thickness $3 \mathrm{~mm}$ ), aluminum foil (0.05 mm thick; Fig. 3A1), a visible range filter (360$760 \mathrm{~nm}$; Fig. 3B1) or a near-infrared range filter $(0.8$ to $2.7 \mu \mathrm{m}$; Fig. 3C1 and 3D1) can be mounted. (Both filters were purchased from Edmond Optics, Barrington, NJ.) First, a black acrylic plate was mounted and the curtain was placed between STO and a student (F). When STO sent his Ki toward her upper body through the black acrylic plate, she did not 

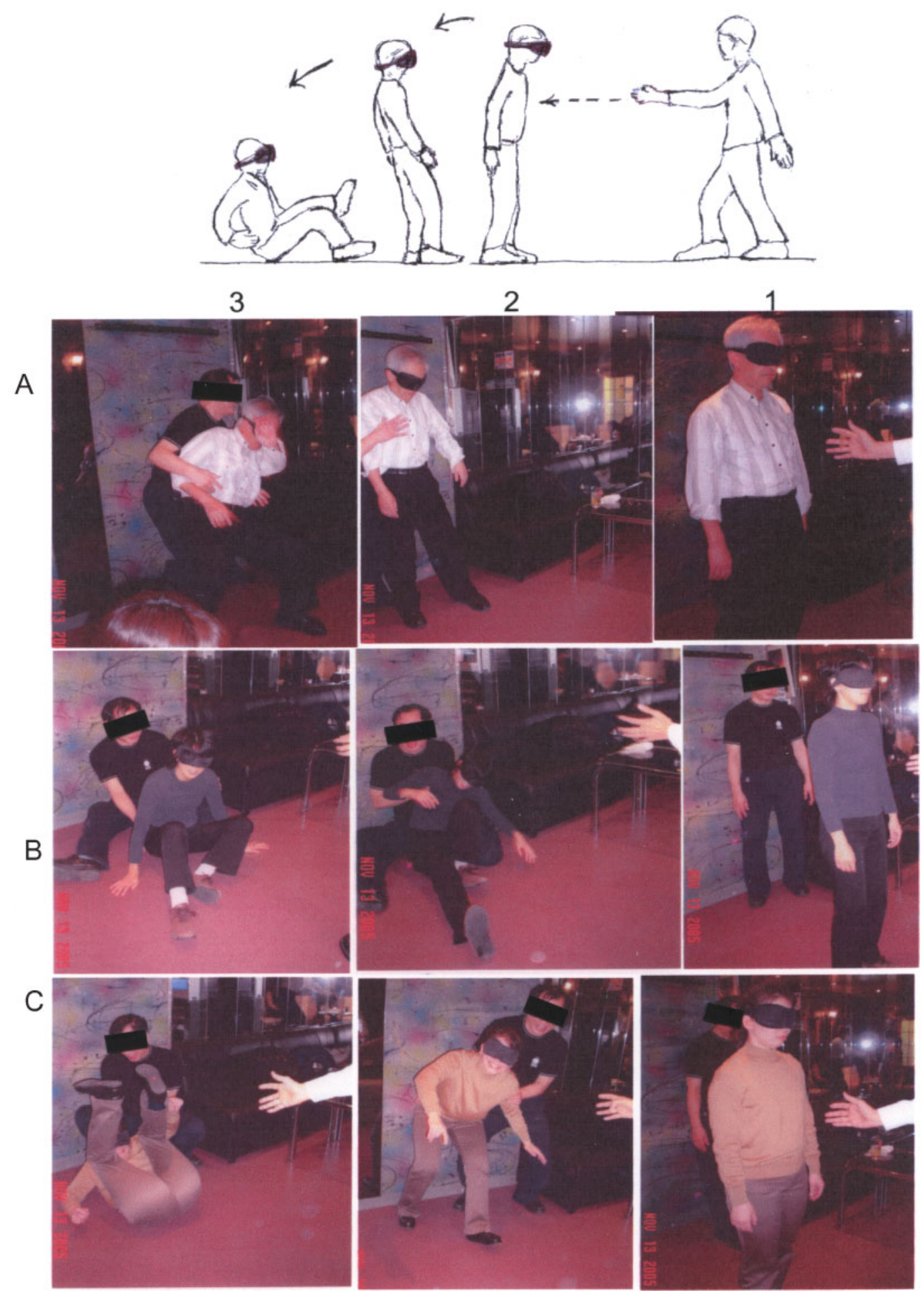

Figure 2. As illustration (top panel) shows a person on the right (STO) sends Ki toward the person on the left. The person on the left (with a blinder and earplugs) stepped backward and fell down. (A) with Mr Y; (B) with Ms F and (C) with Ms M. The events are shown by photos in the sequence of the right side to the left side (1-3).

move (data not shown). When aluminum foil (Figs. 3A1) or a visible range filter (Fig. 3B1) was mounted, the student did not move either (Fig. 3A2 or 3B2). However, when a near-infrared range filter was mounted (Fig. $3 \mathrm{C} 1$ ), the student responded to STO's Ki and fell down (Fig. 3C2).

\section{What Part of the Body Can Sense Ki?}

For this test, the near-infrared range filter was placed in front of the foot of a student (M) as shown in Fig. 3D1. When STO sent his Ki to her foot, she responded and fell down 


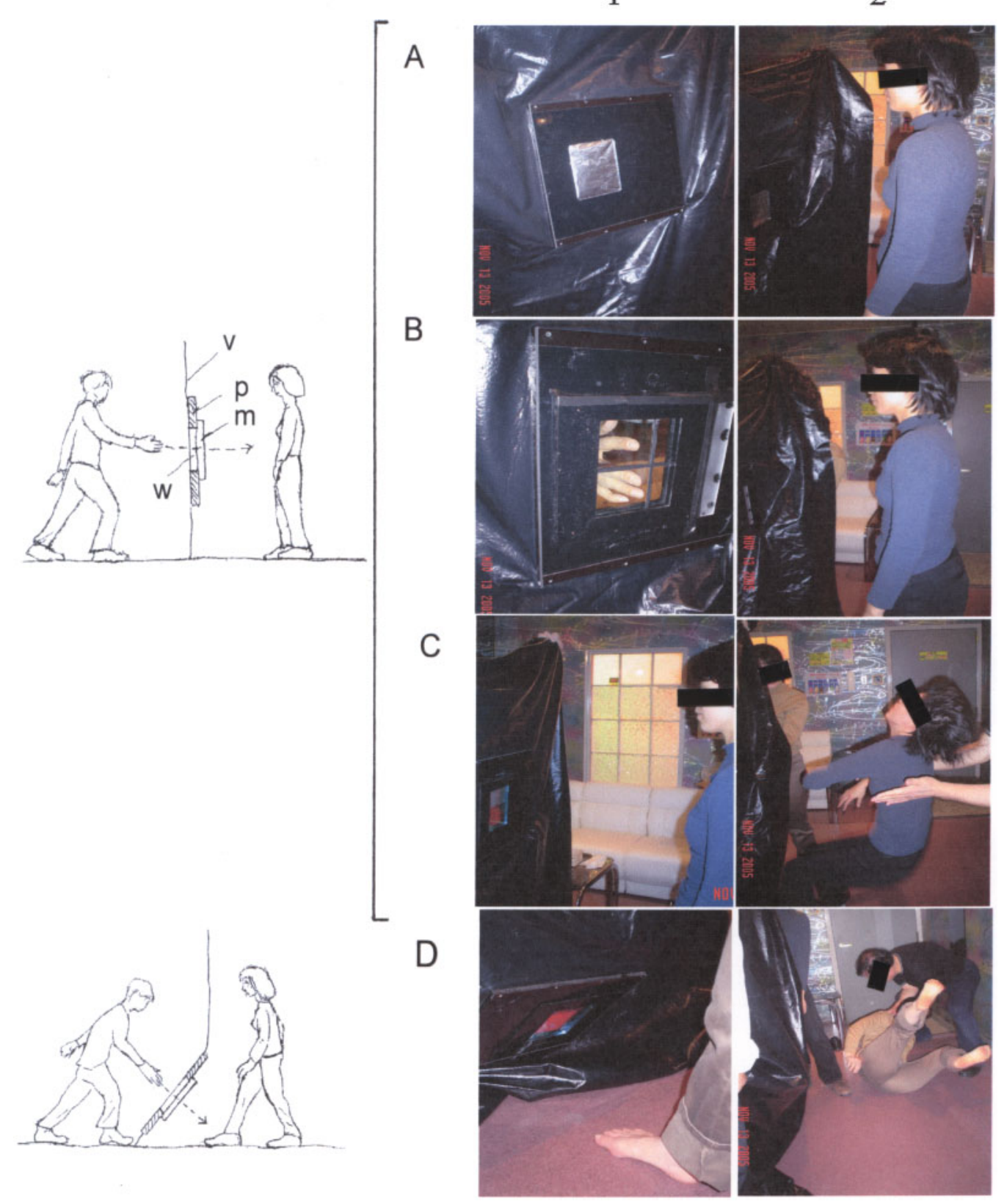

Figure 3. Left top illustration: A person (STO) sent Ki through a window w made in a black acrylic plate $\mathrm{p}$, which is attached to a vinyl curtain v. $\mathrm{m}$ is a material which is being tested for its ability to interrupt $\mathrm{Ki}$ in this experiment. (A1) aluminum foil was placed in front of the upper body of Ms F, (A2) but no response was observed; (B1) a visible range filter $(360-760 \mathrm{~nm})$ in front of Ms F, (B2) but no response was observed; (C1) a near-infrared filter $(0.8-2.7 \mu \mathrm{m})$ in front of Ms F, (C2) who responded and fell down. Left bottom illustration and (D1): STO sent Ki through a near-infrared range filter $(0.8-2.7 \mu \mathrm{m})$ to the right foot of Ms M. (D2) She responded and fell down.

(Fig. 3D2). From this and the above experiments, we know that both upper body and foot can detect $\mathrm{Ki}$.

\section{What Is the Nature of Five Sense-Independent Communication?}

If you simply look at Nishino's Taiki-practice in terms of the student's vigorous movements, you may judge that this is caused by his physical energy, because it looks as if he pushes or throws them using his energy. However, in his class, students sometimes dance, sing or headstand. Nishino shows a gesture for running, stopping or jumping by his hand motions. Even the students who were looking at the opposite direction from him (and in no way could see him) still follow his instructions. This can only be explained by assuming that he sends some information and that the student receives it.

This possibility was tested among students. As shown in Fig. 4A, a student (M) stood on her head facing the other 


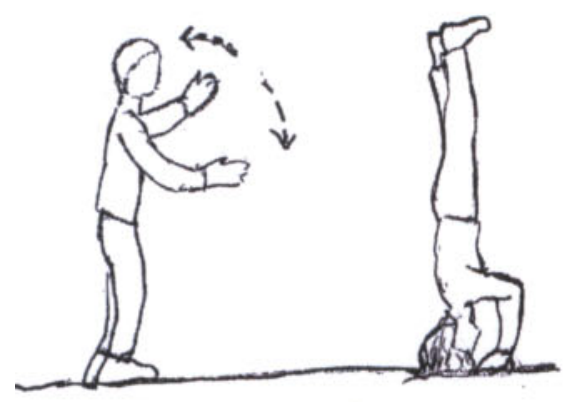

A
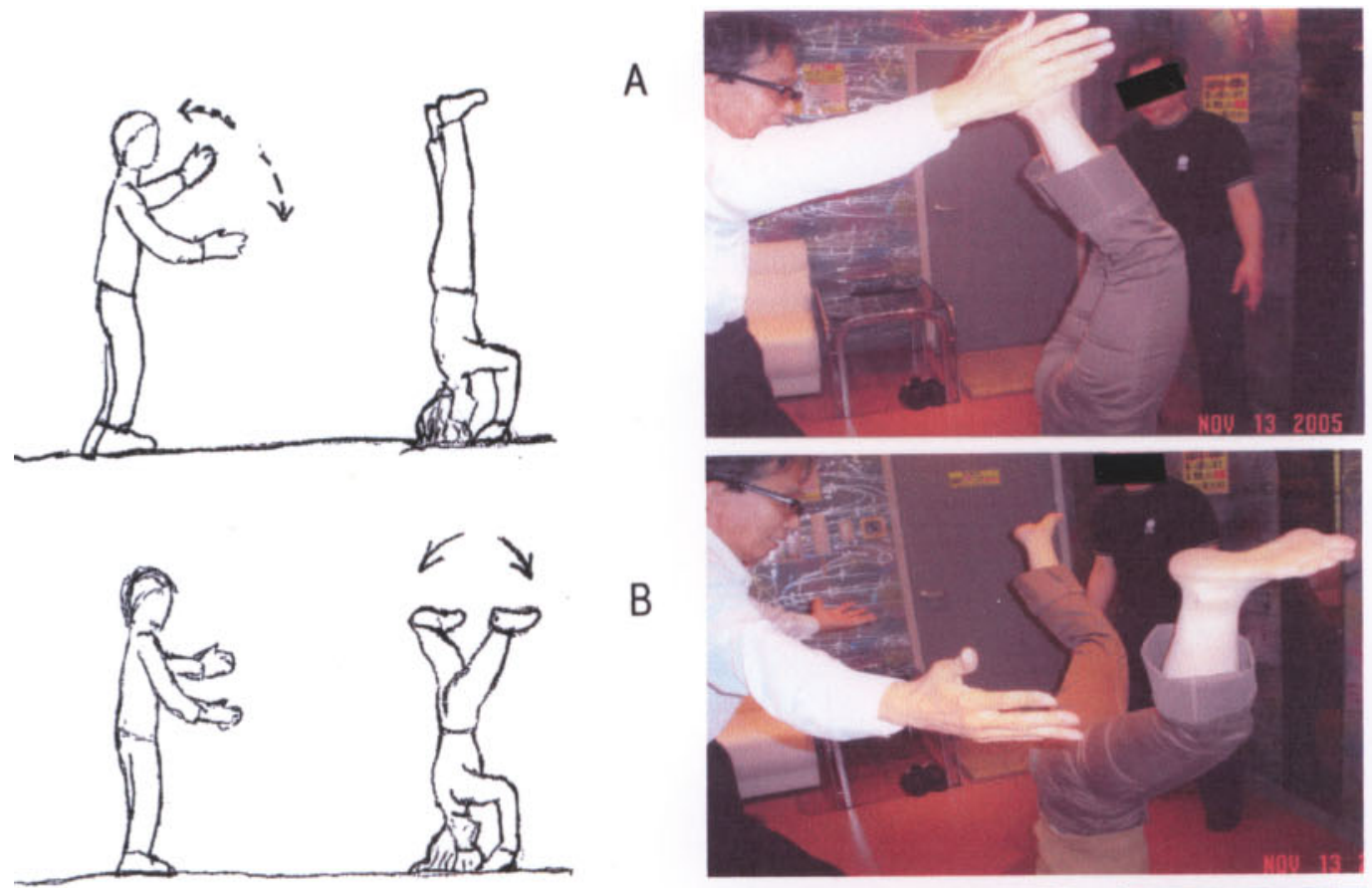

B
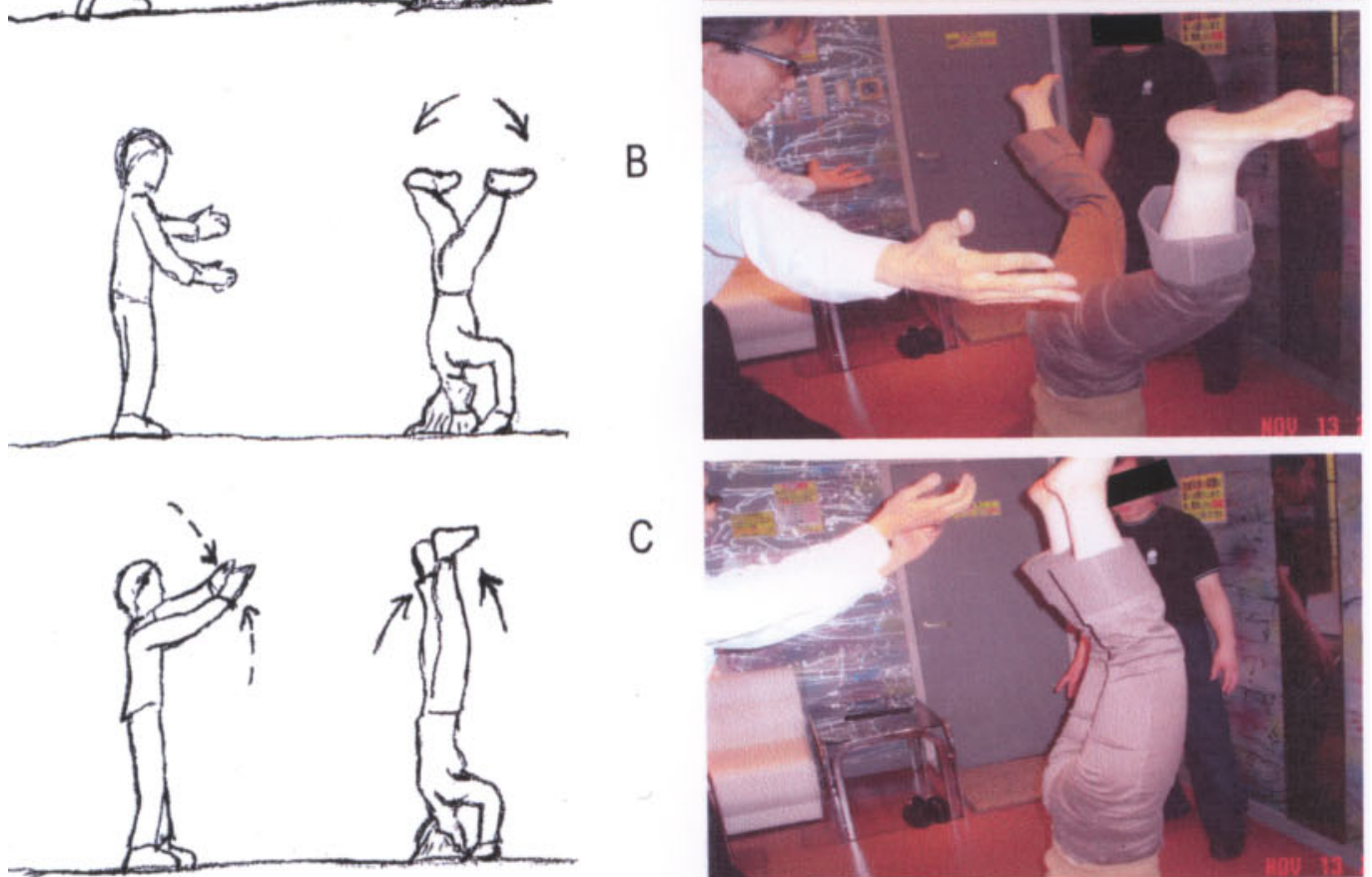

C
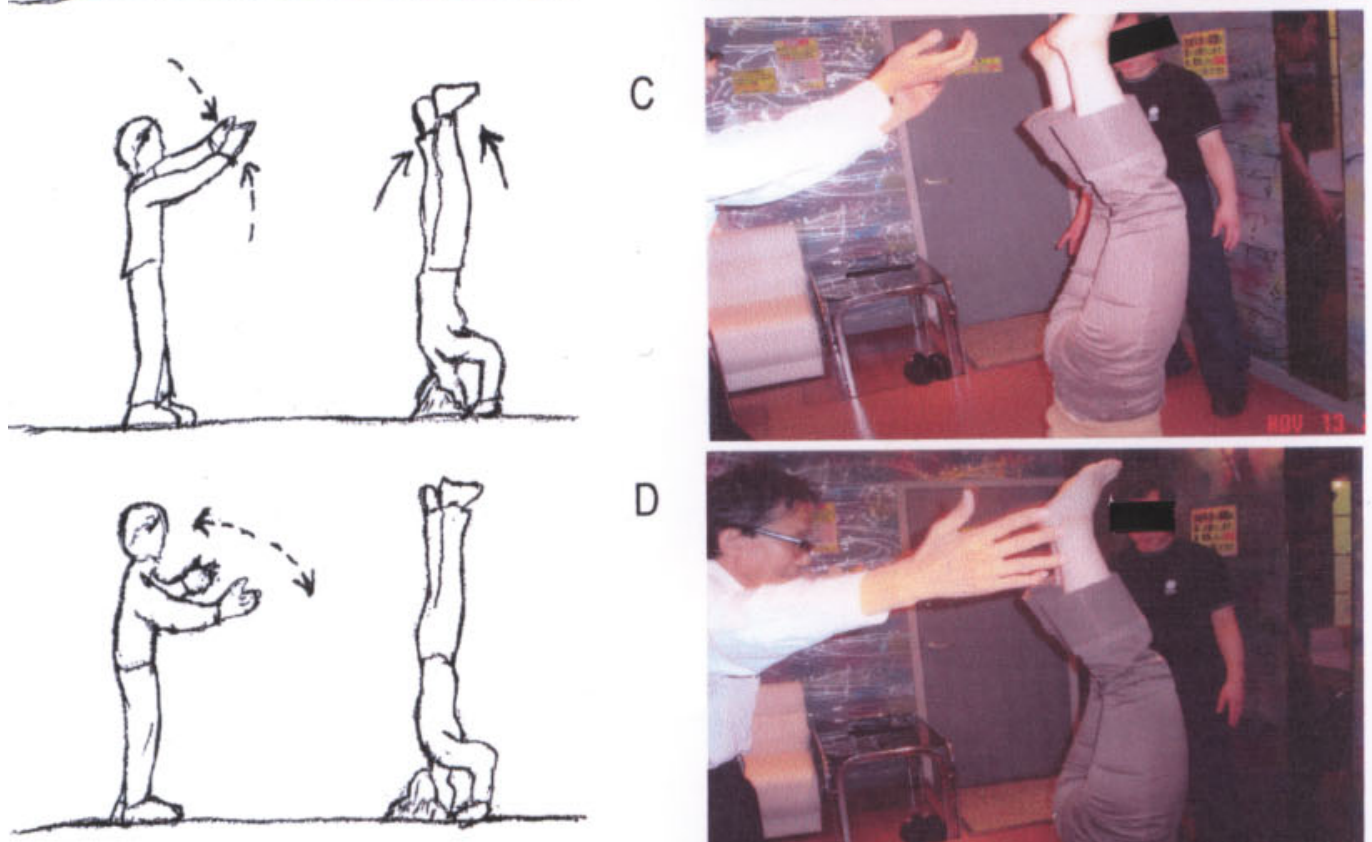

D
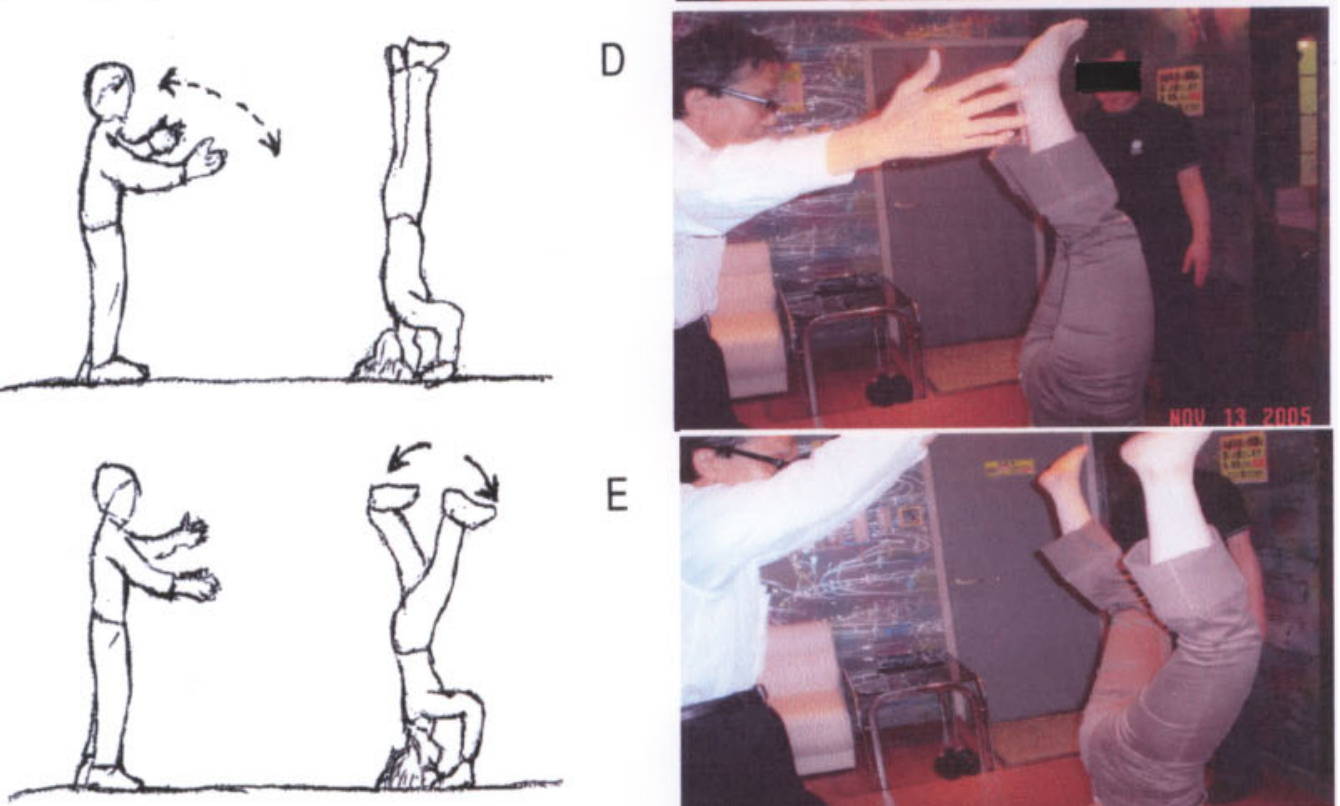

E

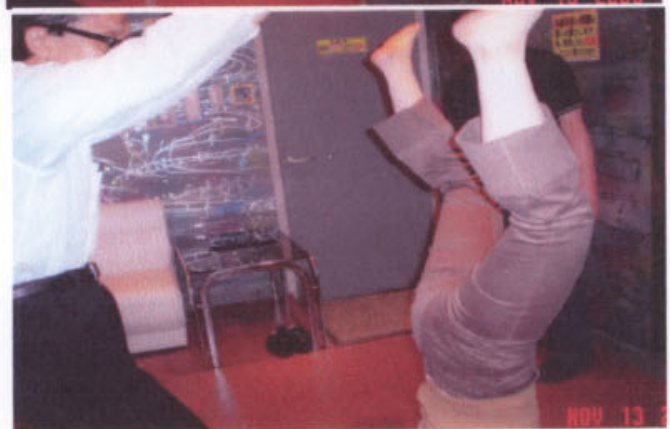

Figure 4. Ms M was standing on her head and facing the opposite direction from where STO stood. STO sent Ki with the order in his mind (A) to open the legs, and (B) Ms M opened her legs. (C) STO sent the order to close the legs and she responded immediately. (D) STO sent the order to open the legs again and (E) she opened her legs. 
direction from where STO stands. When STO concentrates in his mind that she should open her legs (in order to show the camera that he is placing this message in his mind, he opened his hands as seen in Fig. 4B), she responded to it. Subsequently, when he put in his mind that she should close her legs (Fig. 4C) she also responded to it. Fig. 4D shows that STO sent another message to open and Fig. 4E shows that she responded accordingly. Since she was facing the other direction, there was no way that she could watch these signs. When Nishino does this in the class, he can send a message of twisting the legs or bending ankles up and down, to which students also respond. STO tried the same, but $\mathrm{M}$ did not respond to it (photo not shown). Naturally, what a student can do is limited compared to Nishino. However, the above experiment suggests that some messages in STO's mind were transmitted as information, and they were subsequently received by another individual without using the five senses.

\section{Discussion}

Historically, the 'Ki-effect' may have been described as far back as the Biblical era when stories of healing by the extended hand of a special individual were documented. Similar effects of Qi-energy or Qigong therapy were documented in Chinese literature as early as the $3^{\text {rd }}$ and 6th centuries AD (14). In Japanese literature, it first appeared during the Heian Period (8th to 12th century) (8). Actually, a Japanese term for healing is called 'Te-Ate' (literally, 'handtouch'). People knew instinctively that the touch of a hand had a healing effect.

More recently, similar healing techniques were known in Europe as the working of mesmerism or hypnosis. In Asia, the use of life-energy (Qi in Chinese or $\mathrm{Ki}$ in Japanese) in enhancing one's vitality and improving health is employed even today. For example, Qigong therapies are popular in China as Ki-therapies are in Japan.

Unfortunately, these techniques are not well accepted as a branch of today's main-stream sciences, especially in the Western hemisphere. Many people consider them as folk medicine. Some people believe that they are 'supernatural' and 'para-psychological' phenomena. Only a limited number of investigators have been studying them as the object of scientific, medical investigation for the past 30 years.

\section{Nature of Ki in Biological Reactions}

In this article, we emphasized that $\mathrm{Ki}$ (which any individual could learn and develop through the Nishino Breathing Method and the Taiki-practice) is not supernatural. The effects of $\mathrm{Ki}$ can be studied using an established cultured cell model (HepG2) (3). Even Ki emitted by Nishino's students could inhibit cell division (Fig. 1). It was shown that $\mathrm{Ki}$, which is emitted from his fingers, may involve infrared radiation (3). It passes through a near-infrared range filter $(0.8-2.7 \mu \mathrm{m})(7)$. These findings would generate a serious problem that the mind of the observer could change the experimental results. If that is the case, we may need to introduce a 'biological' Uncertainty Principle analogous to Heisenberg's Uncertainty Principle in quantum physics.

\section{How Can Ki Produce Biochemical Effects?}

Machi measured the intensity of infrared radiation from a Qigong healer and estimated it to be $\sim 10 \mu \mathrm{W}(12)$. This leads us to a serious question as to how such a minute amount of energy could trigger tangible biochemical reactions. A possibility may be found in the mechanism of the "cascade reactions' of cellular signal transduction. We have already demonstrated that $\mathrm{Ki}$ decreased the expression of messenger RNA for $c$-myc and increased that for regucalcin in cultured human carcinoma cells, HepG2. Using western blot technique, it was proven that the amount of regucalcin protein indeed increased (3). The mechanism may be explained like this: If a small amount of enzyme $\mathrm{A}$ is expressed by $\mathrm{Ki}$, then its product $\mathrm{B}$ accumulates. If $\mathrm{B}$ happened to be another enzyme or an activator of an enzyme $\mathrm{C}$, then its product $\mathrm{D}$ would accumulate to hundreds of thousands times greater than the amount of $\mathrm{A}$. In cells, many signal transduction mechanisms, consisting of cytokines, receptors, activators and genes, form intimately linked networks to regulate biochemical and genetic reactions. Therefore, a minute change of a component could quickly be amplified millions of times to produce measurable changes in the system.

Although we do not know how infrared radiation can trigger such a change in cellular systems, we could speculate on a possibility. Most bending and stretching vibrations of many chemical bonds can be identified by absorption spectroscopy in a mid-infrared range $(2.5-50 \mu \mathrm{m})$ (Table 1$)$. The overtones (1st harmonics with $1 / 2$ wavelength and 2 nd harmonics with $1 / 3$ wavelength) of these vibrations fall in the near-infrared range. Therefore, it is possible that a particular frequency of $\mathrm{Ki}$ may modulate the vibration of a chemical bond of an enzyme in such a way that it affects its enzymatic activity. If this happens, the microscopic change may be multiplied by the cascading nature of the signal transduction mechanism and result in an observable macroscopic change.

Table 1. Wavelengths $(\mu \mathrm{m})$ of bending and stretching vibrations of some chemical bonds

\begin{tabular}{lll}
\hline Chemical bonds & Bending & Stretching \\
\hline $\mathrm{H}-\mathrm{O}$ & & 3.0 \\
$\mathrm{C}-\mathrm{O}$ & & 9.4 \\
$\mathrm{C}=\mathrm{O}$ & & 5.9 \\
$\mathrm{C}-\mathrm{H}$ & $7.0,14.0$ & $3.0,3.4,3.5$ \\
$\mathrm{C}-\mathrm{N}$ & & 9.3 \\
$\mathrm{C} \equiv \mathrm{N}$ & & 4.5 \\
$\mathrm{C}=\mathrm{C}$ & & $6.3,6.7$ \\
$\mathrm{C} \equiv \mathrm{C}$ & & 4.6 \\
$\mathrm{~N}-\mathrm{H}$ & 6.2 & 2.9 \\
$\mathrm{NO}_{2}$ & & $6.0,6.7$ \\
\hline
\end{tabular}


Table 2. Materials which pass (+) or block (-) Ki related to biochemical reactions and Taiki-reactions

\begin{tabular}{lll}
\hline Materials & $\begin{array}{l}\text { Biochemical } \\
\text { reactions }\end{array}$ & $\begin{array}{l}\text { Taiki- } \\
\text { reactions }\end{array}$ \\
\hline Black acrylic plate & - & - \\
Aluminum foil & - & - \\
Visible range filter $(360-760 \mathrm{~nm})$ & - & - \\
Near IR range filter $(0.8-2.7 \mu \mathrm{m})$ & + & + \\
\hline
\end{tabular}

Thickness: black acrylic plate, $3 \mathrm{~mm}$; aluminum foil, $0.05 \mathrm{~mm}$.

\section{Nature of Ki in Taiki-Practice}

The Ki-effect has no hypnotic basis, because it had an effect on subjects whose eyes were covered and ears were blocked (Fig. 2). Ki, which was emitted from a practitioner and passed through a near-infrared range filter $(0.8-2.7 \mu \mathrm{m})$, was found to cause various Taiki-effects. Namely, when it was aimed at the upper body, it knocked down a student. Even when the $\mathrm{Ki}$ was aimed at the foot of another individual, it still caused a similar response (Fig. 3). When Ki was sent through a near-infrared range filter, the student could not see when and how Ki was sent out, because the filter does not pass visible light. Nevertheless, the students still responded to $\mathrm{Ki}$. This is another proof that the Ki-phenomenon is not a hypnotic effect.

As shown in Table 2, Ki causing biochemical reactions and $\mathrm{Ki}$ causing Taiki-reactions are both blocked by a black acrylic plate, aluminum foil and a visible range filter (360$760 \mathrm{~nm}$ ). However, the Ki for both reactions passes through a near-infrared range filter $(0.8-2.7 \mu \mathrm{m})$. This table suggests that both $\mathrm{Ki}$, which causes biochemical and Taiki-reactions, are one and the same.

How could we explain the Taiki-effects? This could be done as follows: Different parts of the body can detect $\mathrm{Ki}$ (or near-infrared radiation) and send signals to the central nervous system. Then it would dispatch signals to the skeletal muscle to respond. This may be related to the Nishino's idea that all cells in our body can 'sense' Ki. He described each cell as having 'intelligence' (2).

\section{How Can Ki Move Other Individuals?}

Nishino's Taiki-demonstration has been televised several times in Japan over the past 20 years and caused much debate. Many thought this to be fake or a trick, or at best, hypnosis. Strong rejection came from the circle of scientists. They raised the criticism that the human mind cannot produce the huge amount of energy necessary to knock down or throw the opponent. We would like to point out that these scientists 'instinctively' assumed that the energy came from Nishino, and therefore they could not accept what they saw in the demonstration.

In our opinion, the energy came from the opponent. In other words, when the opponent received Nishino's Ki, he/she moved using his/her own energy. If the opponent jumps or falls down using his/her own energy, then this is not a paranormal phenomenon, but a normal phenomenon. This is a physiological reaction triggered by some mechanism, for which we do not currently have scientific explanation.

Two observations may shed light on this and support the interpretation.

(i) At his school, Nishino practices Taiki with several hundred students each day. He does this once with each student everyday (except for new students who have not practiced for 6 months). If he uses his own energy to knock down or throw several hundred students, he must be exhausted. However, after finishing this within 2-3 h, he seems to be not tired. This fact suggests that opponents used their own energy. (ii) The experience of most of the students has been that when they started learning the Nishino Breathing Method, they did not respond much to Nishino's Ki. They just walked back several steps or fell down. However, after practicing it for from several months to a few years, many of them began to respond more vigorously. It may be that the sensitivity of the student toward Nishino's Ki increases with the practice time, and therefore, the more one practices, the more he/she responds. Again, this supports the notion that the students move with their own energy when they receive $\mathrm{Ki}$ from Nishino.

\section{Information Transferred by Ki}

In the Taiki-practice, the most interesting observation is that one can transfer information through Ki. As shown in Fig. 4, when a student was standing on her head facing the other direction and a practitioner sends $\mathrm{Ki}$ signal to open the legs, she opened them. When another $\mathrm{Ki}$ signal to close the legs was sent, she also responded to it.

An important concern in this five sense-independent communication is that the information is not a form of energy. It is a physical quantity called 'entropy' as Shannon described it (15). Energy and entropy are two different physical quantities. The relationship between information and 'negative entropy' in living cells was first pointed out in 1944 by Schrödinger, one of the key figures in the development of quantum physics (16). Of course living beings require energy for their growth, activity and reproduction. However, without information, they do not know how to grow, how to act and how to reproduce. An aspect of entropy in complementary and alternative medicine (CAM) has been presented by Olalde (17).

No matter how strong the Nishino's Ki-energy may be, it alone cannot control the student's movements. His information (in a form of entropy) must be transmitted to students. At his school, he and dozens of instructors show everyday more spectacular examples of the Taiki-practice. Students move vigorously following the instructions they receive. (The information on his class can be found at http://www. nishinojuku.com or can be received by emailing the school at Nishino@gol.com.) This cannot be done by Ki-energy alone, because energy is not an information. For example, the energy 
of a bomb blast can destroy buildings, but it cannot convey information to individuals to open and close their legs.

Shinagawa pioneered the finding that a Qigong healer emitted information $(13,18)$. His group found that the brain wave activity of a volunteer who sat down in front of a Qigong healer synchronized with that of the healer. He also observed that when the healer sent his Qi-energy, the subject lifted her hand (13). He was so surprised to see that information was dispatched by the healer, transmitted through the air and received by another individual. Actually, he had witnessed a 'Taiki-effect.'

Machi observed that infrared radiation (from 0.8 to $25 \mu \mathrm{m}$ ) was emitted from the hand of a Qigong healer, and that its amplitude was modulated with a low frequency of $1.2 \mathrm{~Hz}$ (12). He hypothesized that the information from the healer may be conveyed by the amplitude modulation of the infrared radiation. This is an interesting idea. However, if the information is carried by amplitude modulation of $1.2 \mathrm{~Hz}$, then the time resolution of the information would be on the order of $1.2 \mathrm{~s}$ or longer. In the Nishino's Taiki-practice, his students respond to his instruction almost instantly. When he sends signs to run or to stop, a student responds to it immediately, even though he/she was facing the opposite direction so that he/she could not see Nishino (observers can see both Nishino's gestures and student's responses). If it is not an amplitude modulation, then it may be either frequency modulation or digital modulation. In the past 30 years, many Chinese scientists regarded 'Qi' as a real substance flowing in our body, which can be represented by mass $(m)$. On the contrary, most Japanese scientists treated 'Ki' as energy $(E)$, except for Shinagawa who considered it to be information. We would like to propose that 'Qi' or 'Ki' has both physical quantities of energy $(E)$ and entropy $(S)$. (According to Einstein, both $E$ and $m$ are the same physical entity, because $E=m \mathrm{C}^{2}$.)

\section{Psychological State of Students When They Receive Ki}

When students receive $\mathrm{Ki}$, many of them actively move, scream, dance or sing. They seem to be very vigorous and happy. Toshihiro Nukiwa (professor of Tohoku University School of Medicine) wrote that the Nishino Breathing Method enabled him to encounter the wilderness hidden inside himself (2). In his analysis, the Taiki-practice enables individuals to wake up to their 'archetype' (19). The archetype is the concept in Jungian psychology which lies at the deepest level of an individual's life as fundamental types of life/personality entity. He also recognized that encountering his own identity had an important meaning for him because it gave him a chance to experience new physical sensations and to discover his latent abilities (2).

Yuasa proposed that Ki-phenomena appeared when our deep psychological state surfaced to our conscious mind (8). He suggested that the study of $\mathrm{Ki}$ would bring three fields together, namely, physics, physiology and psychology.
We would like to propose that Taiki-practice may bring us instantly to the level of 'Collective Unconsciousness' (an unconscious state in Jungian psychology which is shared by all human beings regardless of time and ethnic background) (20). It has been discussed that through Zen or Transcendental Meditation, one could explore the state of 'Personal Unconsciousness.' However, even to reach this state, many years of meditation training and practice are required. Further, it would be generally difficult to go deeper than that state. If one can visit the state of Collective Unconsciousness instantly by $\mathrm{Ki}$, this may raise the interesting possibility of exploring the deepest level of unconsciousness and may lead to new psychological and clinical applications.

\section{Conclusion}

We presented data to suggest that the Ki-phenomenon is not paranormal, but a normal phenomenon. When aimed at certain cells or subcellular organelles, Ki-energy had distinctive biological effects. When aimed at other individuals, Ki-energy had a significant effect to induce bodily motions. Similar studies have been reported regarding Chinese Qigong therapy. The key points in Ki-related (and Qi-related) therapy and healing would be (i) what kind of energy effect can Ki produce? and (ii) what kind of information can $\mathrm{Ki}$ transfer between two individuals?

Scientific investigations of $\mathrm{Ki}$ started about 30 years ago, but we still know very little and have so much to learn. We believe that further analysis of both 'Ki-energy' and 'Ki-information' may open up a new horizon in life science to apply to CAM.

\section{Summary}

(i) $\mathrm{Ki}$, which can be developed by the Nishino Breathing Method, is neither a paranormal nor para-psychological phenomenon but is a normal phenomenon. Since it is a normal phenomenon, Ki can be studied by modern scientific methodology.

(ii) Not only Nishino, the founder of the method, but also instructors and his students can emit $\mathrm{Ki}$ from their bodies dependent on their training and ability.

(iii) $\mathrm{Ki}$ inhibited cell division of cultured human cancer cells, and it protected isolated rat liver mitochondria from heat-induced deterioration effects.

(iv) $\mathrm{Ki}$ may consist of several different components, but at least it contains near-infrared radiation between 0.8 and $2.7 \mu \mathrm{m}$.

(v) $\mathrm{Ki}$, which causes biochemical reactions, can pass through a near-infrared range filter $(0.8-2.7 \mu \mathrm{m})$, but is blocked by materials, such as black acrylic, aluminum foil and a visible range filter (360-760 nm). $\mathrm{Ki}$, which causes Taiki-reactions, can pass through the same near-infrared range filter $(0.8-2.7 \mu \mathrm{m})$, but is also blocked by black acrylic, aluminum foil and the same visible range filter $(360-760 \mathrm{~nm})$. 
(vi) During the Taiki-practice, Ki seems to carry information which makes five sense-independent communication possible.

(vii) Different parts of the body can detect both Ki-energy and $\mathrm{Ki}$-information during the Taiki-practice.

(viii) When an individual receives both Ki-energy and Ki-information in the Taiki-practice, his/her state of mind may reach the level of 'Collective Unconsciousness' described in Jungian psychology.

(ix) Both Ki-energy and Ki-information may be involved in the healing effects of $\mathrm{Ki}$. This could be a new subject of study in CAM.

(x) Our deeper understanding of Ki-energy and Ki-information may prompt us to re-examine the Cartesian dualism and to introduce the 'biological' Uncertainty Principle.

\section{Acknowledgments}

We thank Master Kozo Nishino for his instruction. Thanks are also due to Drs M. Yamaguchi and Y. Tsurusaki at University of Shizuoka for their collaboration; students of School of Nishino Breathing Method in Osaka for their assistance; $\mathrm{Mr} \mathrm{M}$. Izawa for his assistance in building experimental device; Mr S. J. Dennis for preparing illustrations and Mr J. Lovasz for arranging photographs.

\section{References}

1. Nishino K. Ki-energy in health. Proceedings of the 4th International Congress on Traditional Asian Medicine, Vol. 1, 1994, 148-70.

2. Nishino K. The Breath of Life: Using the Power of Ki for Maximum Vitality. Tokyo, New York, London: Kodansha International, 1997.
3. Ohnishi ST, Ohnishi T, Nishino K, Tsurusaki Y, Yamaguchi M. Growth inhibition of cultured human carcinoma cells by Ki-energy (Life Energy): scientific study of Ki-effect on cancer cells. Evid Based Complement Altern Med 2005;2:387-93.

4. Kimura H, Nagao F, Tanaka Y, Sakai S, Ohnishi ST, Okumura K. Beneficial effects of the Nishino Breathing Method on the immune activity and stress level. J Altern Complement Med 2005;11:285-91.

5. Nishino K. Nishino Breathing Method (in Japanese). Tokyo: Kodansha, 1987.

6. Nishino K. Nishino Breathing Method: Elevation of Self Energy (in Japanese). Tokyo: Gakushu Kenkyu Pub. Co., 2005.

7. Ohnishi ST, Ohnishi T, Nishino K. Ki-energy (life-energy) protects isolated mitochondria from oxidative injury. Evid Based Complement Altern Med 2006 (in press).

8. Yuasa Y. What is Ki? Tokyo: NHK (Nippon Broadcasting Company) Book Publisher, 1991.

9. Yuasa Y, Takemoto T. New Science and the Science of Ki. In: Yuasa Y, Takemoto T (eds). Tokyo: Seido Publishing Co., 1993.

10. Kiang T. Chinese 'Nature Magazine': Chinese style. Nature 1978;275: 697.

11. Yuasa Y. The Body, Self-Cultivation and Ki-energy. Albany, NY: State University of New York Press, 1993. (translated by S. Nagatomo and M. S. Hull).

12. Machi Y. The Science of Ki (in Japanese). Tokyo: Tokyo Denki University Press, 1993.

13. Shinagawa Y. The Science of Qigong (in Japanese). Tokyo: Kobunsha, 1990.

14. Ikegami S. Wonder of Ki. Tokyo: Kodansha Publishing Co., 1991.

15. Shannon CE. A mathematical theory of communication. Bell Syst Tech J 1948;27:379-423, 623-56.

16. Schrodinger E. What is Life?-The Physical Aspect of the Living Cell. Cambridge: Cambridge University Press, 1944.

17. Olalde JA. The systemic theory of living systems and relevance to CAM. Part I: The theory. Evid Based Complement Altern Med 2005;2:13-8.

18. Kawano K, Koito H, Fujiki T, Shinagawa Y. EEG and topography during Chinese 'Qigong' training. Neurosciences 1990;16:503-8.

19. Nishino K. Nishino Breathing Method: Activation of Life Energy (in Japanese). Tokyo: Kodansha, 2004.

20. Jacobi J. The Psychology of C. G. Jung. New Haven: Yale University Press, 1951. (translated by K. W. Bash).

Received December 6, 2005; accepted January 26, 2006 


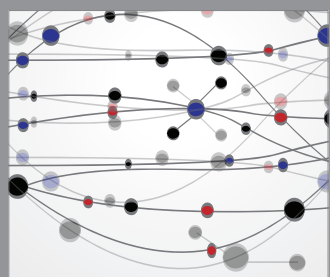

The Scientific World Journal
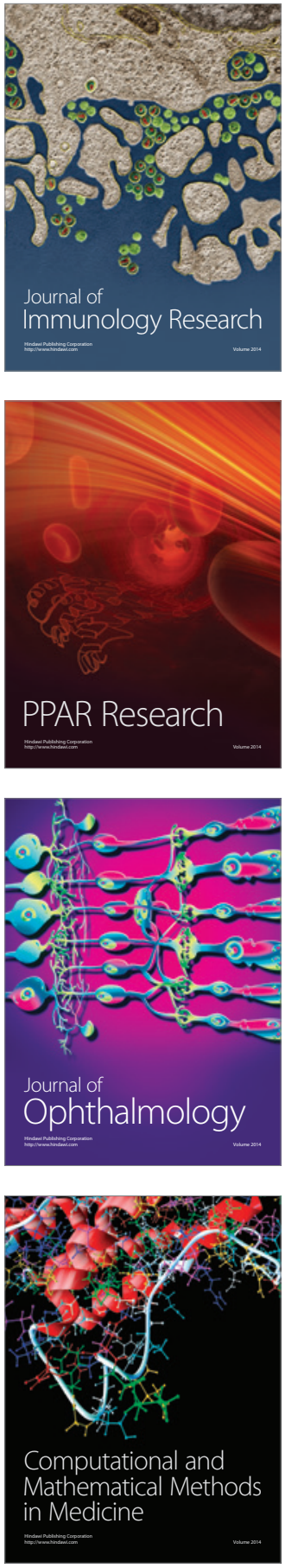

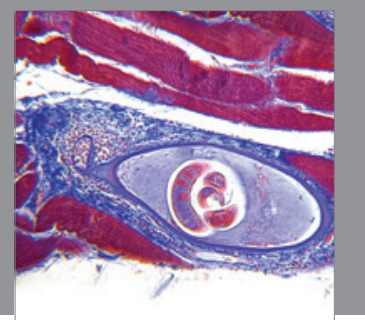

Gastroenterology

Research and Practice
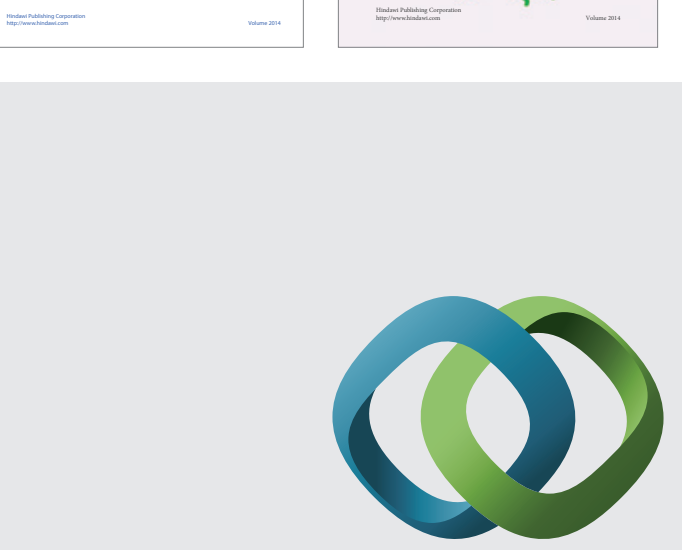

\section{Hindawi}

Submit your manuscripts at

http://www.hindawi.com
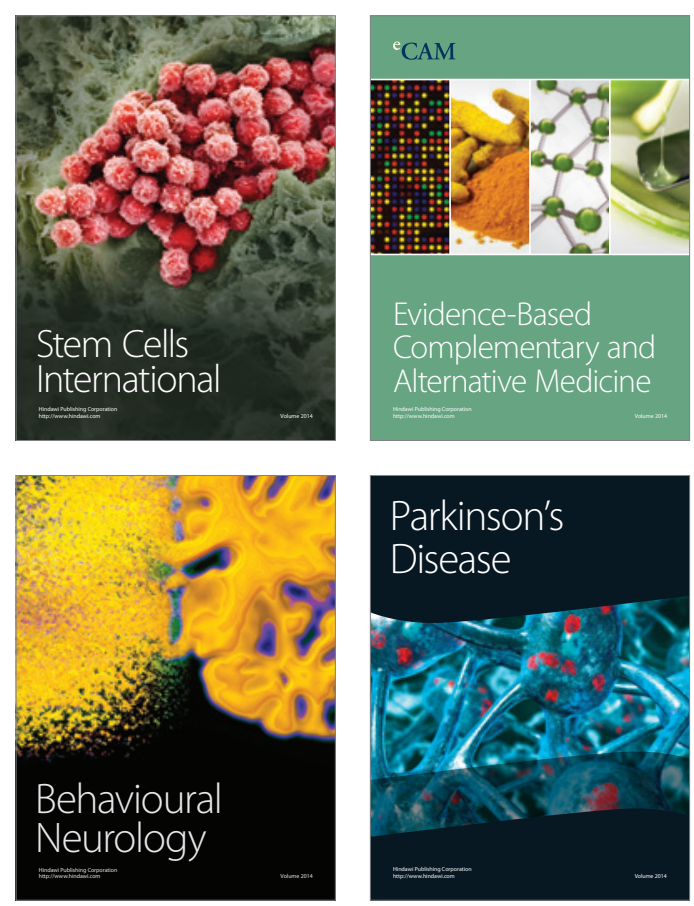

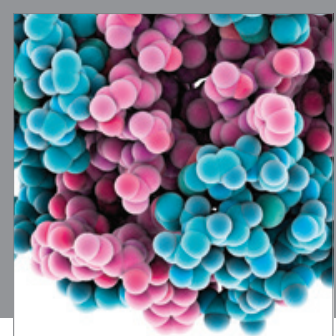

Journal of
Diabetes Research

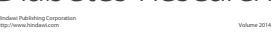

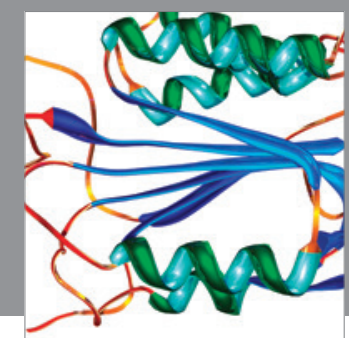

Disease Markers
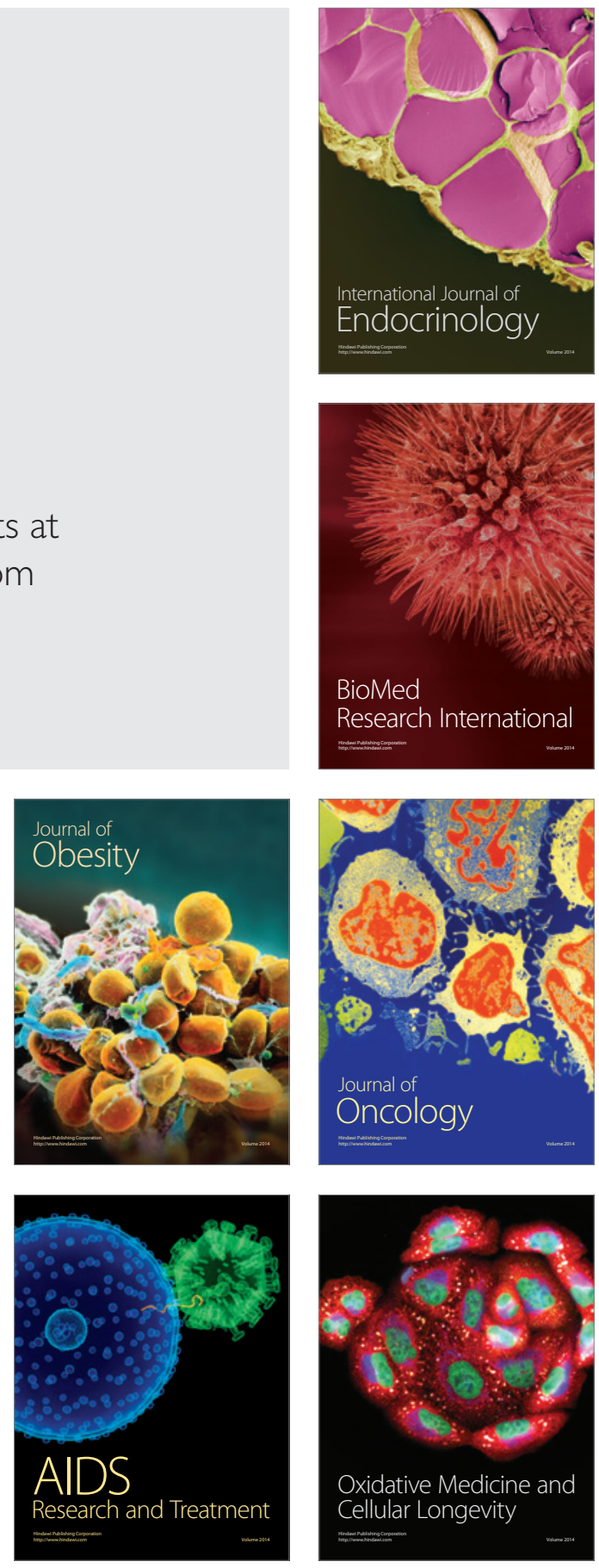